\title{
Existence for Nonlinear Evolution Equations and Application to Degenerate Parabolic Equation
}

\author{
Ning Su and Li Zhang \\ Department of Mathematical Sciences, Tsinghua University, Beijing 100084, China \\ Correspondence should be addressed to Li Zhang; 1-z08@mails.tsinghua.edu.cn
}

Received 6 December 2013; Accepted 5 March 2014; Published 23 April 2014

Academic Editor: Mario Primicerio

Copyright ( 2014 N. Su and L. Zhang. This is an open access article distributed under the Creative Commons Attribution License, which permits unrestricted use, distribution, and reproduction in any medium, provided the original work is properly cited.

\begin{abstract}
We consider an abstract Cauchy problem for a doubly nonlinear evolution equation of the form $(d / d t) \mathscr{A}(u)+\mathscr{B}(u) \ni f(t)$ in $V^{\prime}, t \in(0, T]$, where $V$ is a real reflexive Banach space, $\mathscr{A}$ and $\mathscr{B}$ are maximal monotone operators (possibly multivalued) from $V$ to its dual $V^{\prime}$. In view of some practical applications, we assume that $\mathscr{A}$ and $\mathscr{B}$ are subdifferentials. By using the back difference approximation, existence is established, and our proof relies on the continuity of $\mathscr{A}$ and the coerciveness of $\mathscr{B}$. As an application, we give the existence for a nonlinear degenerate parabolic equation.
\end{abstract}

\section{Introduction}

Let $V$ be a real reflexive Banach space, and let $\mathscr{A}, \mathscr{B}$ be maximal monotone operators (possibly multivalued) from $V$ to its dual $V^{\prime}$. In this paper, we consider the abstract evolution equation:

$$
\begin{gathered}
\frac{d}{d t} \mathscr{A}(u(t))+\mathscr{B}(u(t)) \ni f(t) \quad t \in(0, T], \\
\mathscr{A}(u(0)) \ni v_{0},
\end{gathered}
$$

where $f:[0, T] \rightarrow V^{\prime}$ and $v_{0} \in V^{\prime}$ are given. Inspired by some practical applications (see [1-5]), $\mathscr{A}$ and $\mathscr{B}$ in our work are assumed to be subdifferentials of proper, convex, and lower semicontinuous functions on $V$.

During the past decades, the problem has been investigated in many papers, such as [1, 2,6-16]. For the case that $\mathscr{A}=I$ and $\mathscr{B}$ is a subdifferential operator, the existence and uniqueness in the Hilbert space framework (i.e., $V=H$ ) were established in $[11,13,15,16]$, and the unique solvability in the $V-V^{\prime}$ setting was given by Akagi and Ôtani [2]. Assuming that $\mathscr{A}$ is continuous, $\mathscr{B}$ is continuous and elliptic in some sense, Alt and Luckhaus proved the existence in [8], and Otto established the $L^{1}$-contraction and uniqueness in [17]. For the case that $\mathscr{A}$ is Lipschitz continuous and $\mathscr{B}$ is coercive, the existence theory was given in [14]. In fact, if $\mathscr{A}$ is Lipschitz continuous and invertible, the problem (1) can be rewritten as

$$
\frac{d u}{d t}+\mathscr{B}(v(t)) \ni f(t), \quad v(t) \in \mathscr{A}^{-1}(u(t)), t \in(0, T] .
$$

Under the condition that $\mathscr{A}^{-1}$ is a bi-Lipschitz subdifferential operator, this problem was investigated and solved in [11]. Then the result was extended to a more general case in [12], where $\mathscr{A}^{-1}$ is a maximal monotone operator.

More generally, both $\mathscr{A}$ and $\mathscr{B}$ are possibly nonlinear, and such equations are said to be doubly nonlinear. On the assumption that one of $\mathscr{A}, \mathscr{B}$ is a subdifferential operator and the other is strongly monotone, the existence was established in [10]. In addition, many practical applications (see [1-5]) suggested that both $\mathscr{A}$ and $\mathscr{B}$ are subdifferential operators. For the case that $\mathscr{A}$ and $\mathscr{B}$ are subdifferentials of functions on a Hilbert space, the existence was given in [6]. Supposing that $\mathscr{A}$ is a subdifferential operator in a Hilbert space $H$ and $\mathscr{B}$ is a subdifferential operator in a real reflexive Banach space $V$, respectively, Barbu [9] and Akagi [1,7] obtained the existence with some appropriate assumptions imposed on $\mathscr{B}$.

In some papers (such as $[6,11,12]$ ), the problems investigated are time dependent; that is, $\mathscr{B}$ (possibly together with $\mathscr{A})$ is time dependent. In this paper, we aim to extend the first existence theory in [14] to the case that $\mathscr{A}$ is only continuous 
but not Lipschitz continuous, and $\mathscr{B}$ is coercive with some other appropriate conditions. Our basic assumptions and the existence theory are stated in Section 2, and the preliminaries are introduced in Section 3. In Section 4, we use the backward difference quotient to approximate the time derivative as in [8] and solve the problem by means of convex analysis and uniform estimation, in which we make good use of the properties of subdifferentials and maximal monotone operator. In Section 5, as an application of the abstract existence theorem, we give the existence for a nonlinear degenerate parabolic equation.

\section{Basic Assumptions and Existence Theorem}

2.1. Basic Assumptions. To state our assumptions clearly, we introduce some notations.

Let $X$ be a real reflexive Banach space and let $\mathscr{F}(X)$ be the set of all proper, convex, and lower semicontinuous functions $\psi: X \rightarrow(-\infty,+\infty]$, where proper means that $\psi \not \equiv+\infty$.

For any $\psi \in \mathscr{F}(X)$, its subdifferential of $\psi$ at $u$, denoted by $\partial_{X} \psi(u)$, is given by

$$
\begin{aligned}
\partial_{X} \psi & (u) \\
& =\left\{v \in X^{\prime}:\langle v, x-u\rangle \leq \psi(x)-\psi(u), \forall x \in D(\psi)\right\},
\end{aligned}
$$

where $D(\psi)=\{x \in X: \psi(x)<+\infty\}$. Then, we define the subdifferential operator $\partial_{X} \psi: X \rightarrow 2^{X^{\prime}} ; u \mapsto \partial_{X} \psi(u)$ with the domain $D\left(\partial_{X} \psi\right)=\left\{x \in D(\psi): \partial_{X} \psi(x) \neq \varnothing\right\}$.

Let $V$ be a real reflexive Banach space, and let $H$ be a real Hilbert space, where $V$ is densely and compactly embedded in $H$. Denote the injection by $i: V \rightarrow H$.

Our basic assumptions are as follows.

(A) $\mathscr{A}=\partial_{V}\left(\Phi_{A} \circ i\right)$, where $\Phi_{A} \in \mathscr{F}(H), \Phi_{A}(0) \leq 0$, and $\partial_{H} \Phi_{A}$ is continuous.

(B) $\mathscr{B}=\partial_{V} \Phi_{B}$, where $\Phi_{B} \in \mathscr{F}(V)$ with $D\left(\partial_{V} \Phi_{B}\right) \neq \varnothing$, satisfying that

(B1) $\Phi_{B}$ is coercive; that is,

$$
\lim _{\|u\|_{V} \rightarrow+\infty} \frac{\Phi_{B}(u)}{\|u\|_{V}}=+\infty ;
$$

(B2) there exists a nondecreasing function $l(\cdot): \mathbb{R} \rightarrow$ $\mathbb{R}$, such that

$\|v\|_{V^{\prime}} \leq l\left(\Phi_{B}(u)\right), \quad \forall u \in D\left(\partial_{V} \Phi_{B}\right), \forall v \in \partial_{V} \Phi_{B}(u)$.

(F) $f \in W^{1,2}\left(0, T ; V^{\prime}\right)$.

(V) There exists $u_{0} \in \operatorname{int}(D(\mathscr{A})) \bigcap D(\mathscr{B})$ such that $v_{0}=$ $\mathscr{A}\left(u_{0}\right)$.

Remark 1. (1) Condition (A) implies that the operator $A$ is single valued.

(2) $\Phi_{A}(0) \leq 0$ is equivalent to $\Phi_{A}(0)<+\infty$.

(3) (B1) implies the coerciveness of $\mathscr{B}$; that is,

$$
\lim _{\substack{\|u\|_{V} \rightarrow+\infty \\ v \in \mathscr{B}(u)}} \frac{\langle v, u\rangle}{\|u\|_{V}}=+\infty .
$$

\subsection{Existence Theorem}

Theorem 2. Assume (A), (B), (F), and (V) are all satisfied. Then, there exists at least one solution triplet

$$
\begin{gathered}
u \in L^{\infty}(0, T ; V), \quad v \in L^{\infty}\left(0, T ; V^{\prime}\right) \cap W^{1,2}\left(0, T ; V^{\prime}\right), \\
w \in L^{\infty}\left(0, T ; V^{\prime}\right),
\end{gathered}
$$

such that $u$ is a solution of (1); that is, $v(t)=\mathscr{A}(u(t)), w(t) \in$ $\mathscr{B}(u(t))$, and

$$
\frac{d}{d t} v(t)+w(t)=f(t)
$$

Moreover, $v(t) \rightarrow v_{0}$ strongly in $V^{\prime}$ as $t \rightarrow 0$.

\section{Preliminaries}

The proofs related to this section can be found in $[3,18-23]$.

\subsection{Lower Semicontinuous Functions}

Lemma 3. Let $\psi \in \mathscr{F}(X)$. Then $\psi$ is bounded from below by an affine function; that is, there exist $x_{0}^{*} \in X^{\prime}$ and $\beta \in \mathbb{R}$ such that

$$
\psi(x) \geq\left\langle x_{0}^{*}, x\right\rangle+\beta, \quad \forall x \in X .
$$

Let $\psi$ be a function from $X$ to $(-\infty,+\infty]$, then its conjugate function $\psi^{*}$, originally developed by Fenchel, is defined as

$$
\psi^{*}(v):=\sup \{\langle v, u\rangle-\psi(u): u \in X\}, \quad v \in X^{\prime} .
$$

Lemma 4. Let $X$ be a real reflexive Banach space.

(1) For any $\psi: X \rightarrow(-\infty,+\infty], \psi^{*}$ is convex and lower semicontinuous.

(2) For any $\psi \in \mathscr{F}(X), \psi^{*}$ is proper.

Remark 5. Let $\psi \in \mathscr{F}(X)$ with $\psi(0) \leq 0$. Then, $\psi^{*}(v) \geq 0$ for any $v \in D\left(\psi^{*}\right)$. In fact, from the definition of $\psi^{*}$,

$$
\psi^{*}(v)=\sup \{\langle v, u\rangle-\psi(u): u \in X\} \geq-\psi(0) \geq 0 .
$$

3.2. Maximal Monotone Operators. Let $X$ be a real reflexive Banach space. An operator $\mathscr{T}: X \rightarrow 2^{X^{\prime}}$ is called monotone, if

$$
\begin{aligned}
& \left\langle v_{1}-v_{2}, u_{1}-u_{2}\right\rangle \geq 0, \\
& \forall u_{j} \in D(\mathscr{T}), \quad \forall v_{j} \in \mathscr{T}\left(u_{j}\right), \\
& \quad j=1,2,
\end{aligned}
$$

where $D(\mathscr{T})=\{x \in X: \mathscr{T}(x) \neq \varnothing\}$. In addition, $\mathscr{T}$ is called maximal if it has no proper monotone extension in $X$; that is, for any $x \in X$ and any $y \in X^{\prime}$,

$$
\langle v-y, u-x\rangle \geq 0, \quad \forall u \in D(\mathscr{T}), v \in \mathscr{T}(u)
$$

only if $y \in \mathscr{T}(x)$. 
Lemma 6. Let $X$ be a real reflexive Banach space, and let $\mathscr{T}$ : $X \rightarrow 2^{X^{\prime}}$ be maximal monotone. Let $x_{i} \in X$ and $y_{i} \in \mathscr{T}\left(x_{i}\right)$ be such that $x_{i} \rightarrow x, y_{i} \rightarrow y$, and $\lim \sup \left\langle y_{i}, x_{i}\right\rangle \leq\langle y, x\rangle$. Then $y \in \mathscr{T}(x)$.

Lemma 7. Let $X$ be a reflexive Banach space. Let $\mathscr{T}_{1}, \mathscr{T}_{2}$ : $X \rightarrow 2^{X^{\prime}}$ be maximal monotone operators such that $\operatorname{int}\left(D\left(\mathscr{T}_{1}\right)\right) \cap D\left(\mathscr{T}_{2}\right) \neq \varnothing$. Then $\mathscr{T}_{1}+\mathscr{T}_{2}$ is maximal monotone from $X$ to $2^{X^{\prime}}$.

Lemma 8. Let $\mathscr{T}_{1}, \mathscr{T}_{2}: X \rightarrow 2^{X^{\prime}}$ be maximal monotone operators such that

(a) $\mathscr{T}_{2}$ is regular; that is, for all $x \in D\left(\mathscr{T}_{2}\right)$ and all $y \in$ $R\left(\mathscr{T}_{2}\right)$, we have

$\sup \left\{\langle v-y, x-u\rangle: u \in X, v \in \mathscr{T}_{2}(u)\right\}<+\infty$;

(b) $D\left(\mathscr{T}_{1}\right) \bigcap D\left(\mathscr{T}_{2}\right) \neq \varnothing$ and $R\left(\mathscr{T}_{2}\right)=X^{\prime}$;

(c) $\mathscr{T}_{1}+\mathscr{T}_{2}$ is maximal monotone.

Then, $R\left(\mathscr{T}_{1}+\mathscr{T}_{2}\right)=X^{\prime}$.

3.3. Subdifferentials. For any $\psi \in \mathscr{F}(X)$, its subdifferential $\partial_{X} \psi$, defined as (3), has the following properties.

Lemma 9. Let $X$ be a reflexive Banach space and $\psi \in \mathscr{F}(X)$. Then,

(1) $\partial_{X} \psi: X \rightarrow 2^{X^{\prime}}$ is maximal monotone;

(2) $v \in \partial_{X} \psi(u) \Leftrightarrow u \in \partial_{X^{\prime}} \psi^{*}(v) \Leftrightarrow \psi(u)+\psi^{*}(v)=\langle v, u\rangle$.

Lemma 10. Let $X$ be a reflexive Banach space and $A=\partial_{X} \psi$, where $\psi \in \mathscr{F}(X)$. Then the following conditions are equivalent:

$$
\begin{gathered}
\lim _{\substack{\|\|_{V} \rightarrow+\infty \\
x \in D(\psi)}} \frac{\psi(x)}{\|x\|_{V}}=+\infty ; \\
R(A)=X^{\prime}, \quad A^{-1} \text { is bounded. }
\end{gathered}
$$

Let $T>0$ and $1<p<+\infty$, and let $\psi$ be a function on $[0, T] \times X$ such that

(1) there are two constants $\alpha$ and $\beta$, for all $x \in V$ and all $t \in[0, T]$,

$$
\psi(t, x)+\alpha\|x\|_{X}+\beta \geq 0
$$

(2) $\psi(t, \cdot) \in \mathscr{F}(X)$ for each $t \in[0, T]$ and the function $t \rightarrow \psi(t, v(t))$ is measurable for each $v \in L^{p}(0, T ; X)$.

Then, we can define a function $\mathscr{F}_{\psi}$ on $L^{p}(0, T ; X)$ as follows:

$$
\mathscr{F}_{\psi}(u)= \begin{cases}\int_{0}^{T} \psi(t, u(t)) d t, & \text { if } \psi(\cdot, u(\cdot)) \in L^{1}(0, T), \\ +\infty, & \text { otherwise }\end{cases}
$$

which is proper, convex, and lower semicontinuous and $\mathscr{F}_{\psi}>$ $-\infty$ on $L^{p}(0, T ; X)$. For any $u \in L^{p}(0, T ; X)$, we call $v \in$ $\partial \mathscr{F}_{\psi}(u)$ in the sense of $L^{p^{\prime}}\left(0, T ; X^{\prime}\right)$ if $v \in L^{p^{\prime}}\left(0, T ; X^{\prime}\right)$ and $v \in \partial_{Y} \mathscr{F}_{\psi}(u)$, where $(1 / p)+\left(1 / p^{\prime}\right)=1$ and $Y=L^{p}(0, T ; X)$. Then, we have the following conclusion (Proposition 1.1 of [24]).

Lemma 11. Assume that for each $t \in[0, T]$ and each $z \in X$ with $\psi(t, z)<\infty$, there exists a function $v \in L^{p}(0, T ; X)$ such that $v(t)=z, \psi(\cdot, v(\cdot)) \in L^{1}(0, T), v$ is right-continuous at $t$, and

$$
\limsup _{s \downarrow t} \psi(s, v(s)) \leq \psi(t, z) .
$$

Let $u$ be a function in $L^{p}(0, T ; X)$ such that $\psi(\cdot, u(\cdot)) \in L^{1}(0, T)$ and let $f$ be a function in $L^{p^{\prime}}\left(0, T ; X^{\prime}\right)$. Then, $f \in \partial \mathscr{F}_{\psi}(u)$ in the sense of $L^{p^{\prime}}\left(0, T ; X^{\prime}\right)$, if and only if $f(t) \in \partial_{X} \psi(u(t))$ for a.e. $t \in[0, T]$.

Remark 12. Assume $T>0$ and $\psi \in \mathscr{F}(X)$. Let $u \in L^{2}(0, T ; X)$ and $v \in L^{2}\left(0, T ; X^{\prime}\right)$. Then, $v \in \partial \mathscr{F}_{\psi}(u)$ in the sense of $L^{2}\left(0, T ; X^{\prime}\right)$, if and only if $v(t) \in \partial_{X} \psi(u(t))$ for a.e. $t \in[0, T]$.

Next, we introduce some chain rules of subdifferentials in different forms.

By the definition of $\psi^{*}$ and Lemma 9, we can easily verify the following chain rule in the form of difference quotient.

Lemma 13. Let $v(t) \in \partial_{X} \psi(u(t))$. Then, for each $h>0$,

$$
\begin{aligned}
& \partial_{-h} \psi(u(t)) \leq\left\langle v(t), \partial_{-h} u(t)\right\rangle, \\
& \partial_{-h} \psi^{*}(v(t)) \leq\left\langle\partial_{-h} v(t), u(t)\right\rangle,
\end{aligned}
$$

where $\partial_{-h}$ denotes the backward difference operator,

$$
\partial_{-h} u(t)=\frac{u(t)-u(t-h)}{h} .
$$
[25].

The following chain rule of integral form was proved in

Lemma 14. Assume $p \in[1,+\infty)$, and $(1 / p)+(1 / q)=1$. Let $X$ be a real reflexive Banach space and $\psi \in \mathscr{F}(X)$. Let $u \in W^{1, p}(0, T ; X)$ and $w \in L^{q}\left(0, T ; X^{\prime}\right)$ be such that $w(t) \in$ $\partial_{X} \psi(u(t))$ almost everywhere in $(0, T)$. Then $t \mapsto \psi(u(t))$ is absolutely continuous, and for all $v \in L^{q}\left(0, T ; X^{\prime}\right)$ with $v(t) \in \partial_{X} \psi(u(t))$ almost everywhere in $(0, T)$,

$$
\psi(u(t))-\psi(u(s))=\int_{s}^{t}\left\langle v, \frac{d u}{d t}\right\rangle \quad \forall 0 \leq s \leq t \leq T .
$$

Lemma 15. Let $X$ be a real reflexive Banach space and let $Y$ be a Hilbert space with $X$ densely and compactly embedded in $Y$. Let $\Lambda: X \rightarrow Y$ be a linear continuous operator, and assume that $\psi \in \mathscr{F}(Y)$ is continuous at some point of $R(\Lambda)$ (the range of $\Lambda)$. Then

$$
\partial_{X}(\psi \circ \Lambda)=\Lambda^{\prime} \circ \partial_{Y} \psi \circ \Lambda: X \rightarrow 2^{X^{\prime}},
$$

where $L^{\prime}$ is the dual operator of $\Lambda$. 
Remark 16. Since $\partial_{H} \Phi_{A}$ is continuous and $V$ is densely and compactly embedded in $H, \mathscr{A}=\partial_{V}\left(\Phi_{A} \circ i\right)=i^{\prime} \circ \partial_{H} \Phi_{A} \circ i$ is compact, where $i$ is the injection from $V$ to $H$.

Lemma 17. Assume (A), (B), and (V) are satisfied. Then $\mathscr{A}+\mathscr{B}$ is maximal monotone from $V$ to $2^{V^{\prime}}$ and $R(\mathscr{A}+\mathscr{B})=V^{\prime}$.

Proof. Since $\operatorname{int}(D(\mathscr{A})) \bigcap D(\mathscr{B}) \neq \varnothing$, we get the maximal monotonicity of $\mathscr{A}+\mathscr{B}$ from Lemmas 9 and 7 .

To prove $R(\mathscr{A}+\mathscr{B})=V^{\prime}$, we only need to verify that $\mathscr{A}$ and $\mathscr{B}$ satisfy Lemma 8. Applying (4) and Lemma 10, we can deduce that $R(\mathscr{B})=V^{\prime}$, and then the proof is completed if we could show that $\mathscr{B}=\partial_{V} \Phi_{B}$ is regular.

In fact, for any real reflexive Banach space $X$ and any $\psi \in$ $\mathscr{F}(X), \partial_{X} \psi$ is regular.

Take $x \in D\left(\partial_{X} \psi\right)$ and $y \in R\left(\partial_{X} \psi\right)$. Since $R\left(\partial_{X} \psi\right) \subset$ $D\left(\psi^{*}\right)$,

$$
\begin{aligned}
\langle v-y, x-u\rangle & \leq \psi(x)-\psi(u)-\langle y, x-u\rangle \\
& \leq \psi(x)+\psi^{*}(y)-\langle y, x\rangle
\end{aligned}
$$

holds for any $u \in D(\psi)$ and for any $v \in \partial_{X} \psi(u)$. Since the right-hand side of (23) is a constant independent of $v$ and $u$,

$$
\sup \left\{\langle v-y, x-u\rangle: u \in D\left(\partial_{X} \psi\right), v \in \partial_{X} \psi(u)\right\}<+\infty \text {; }
$$

that is, $\partial_{X} \psi$ is regular.

\section{Proof of Theorem 2}

In this section, to prove Theorem 2, we use the backward difference to approximate the time derivative. Since we can establish the solvability of the resulting approximate equations from Lemma 17, then, combining convex analysis and uniform estimation, we verify the existence.

4.1. Approximate Problems and Approximate Solutions. Let $N$ be a positive integer, and $h=T / N$.

To prove Theorem 2, we approximate the time derivative in (1) by $\partial_{-h}$ and approximate $f$ by $f_{h}$ :

$$
\begin{aligned}
f_{h}(t)= & \frac{1}{h} \int_{(k-1) h}^{k h} f(s) d s, \\
& (k-1) h<t \leq k h, \quad k=1,2, \ldots, N .
\end{aligned}
$$

These lead to the approximate problem

$$
\begin{gathered}
\partial_{-h} \mathscr{A}(u(t))+\mathscr{B}(u(t)) \ni f_{h}(t) \quad \text { in } V^{\prime}, t \in(0, T] \\
\mathscr{A}\left(u_{h}(t)\right)=v_{0}, \quad t \in(-h, 0]
\end{gathered}
$$

and we can solve the solution $u_{h}(t)$ inductively for $t \in$ $(-h, k h], k=0,1, \ldots, N$, as follows.
For $t \in(-h, 0]$, we set $u_{h}(t)=u_{0}, v_{h}(t)=v_{0}=\mathscr{A}\left(u_{0}\right)$. Suppose that we have a solution of (26) with $T=k h, 0 \leq k<$ $N$, which implies that we have a solution triplet

$$
\begin{gathered}
u_{h}(t) \in V, \quad v_{h}(t)=\mathscr{A}\left(u_{h}(t)\right), \\
w_{h}(t) \in \mathscr{B}\left(u_{h}(t)\right), \\
t \in(-h, k h] .
\end{gathered}
$$

Consider the problem (26) with $T=(k+1) h$, which is equivalent to

$$
\begin{array}{r}
\mathscr{A}\left(u_{h}(t)\right)+h \mathscr{B}\left(u_{h}(t)\right) \ni h f_{h}(t)+v_{h}(t-h) \text { in } V^{\prime}, \\
-h<t \leq(k+1) h .
\end{array}
$$

Since for $t \in(k h,(k+1) h], h f_{h}(t)+v(t-h) \in V^{\prime}$, by Lemma 17, this problem has at least one solution $u_{h}(t)$ for $t \in(k h,(k+$ $1) h]$. Then we can solve $u_{h}(t)$ inductively for $t \in(-h, k h]$, $k=0,1, \ldots, N$, and consequently the problem (26) has at least one solution triplet:

$$
\begin{gathered}
u_{h}(t) \in V, \quad v_{h}(t)=\mathscr{A}\left(u_{h}(t)\right), \\
w_{h}(t) \in \mathscr{B}\left(u_{h}(t)\right),
\end{gathered}
$$

such that

$$
\begin{gathered}
\partial_{-h} v_{h}(t)+w_{h}(t)=f_{h}(t) \quad \text { in } V^{\prime}, \quad 0<t \leq T, \\
v_{h}(0)=v_{0} .
\end{gathered}
$$

Obviously, the triplet $\left(u_{h}(t), v_{h}(t), w_{h}(t)\right)$ is piecewise constant; that is, the triplet is constant in each interval $((k-$ 1) $h, k h], k=0,1, \ldots, N$.

In the following, we aim to obtain some uniform estimates on the approximation solutions (see Section 4.2) and then solve the problem (1) by taking the limit of an appropriate subsequence (see Section 4.3).

\subsection{Uniform Estimates}

Lemma 18. There exists a constant $C>0$, such that

$$
\begin{aligned}
& \sup _{0 \leq t \leq T}\left\|u_{h}(t)\right\|_{V} \leq C, \\
& \sup _{0 \leq t \leq T}\left\|w_{h}(t)\right\|_{V^{\prime}} \leq C, \\
& \left\|\partial_{-h} v_{h}\right\|_{L^{2}\left(0, T ; V^{\prime}\right)} \leq C .
\end{aligned}
$$

Proof. Applying (30), for any $\tau=k h, 1 \leq k \leq N$, we have

$$
\begin{gathered}
\int_{0}^{\tau}\left\langle\partial_{-h} v_{h}(t), \partial_{-h} u_{h}(t)\right\rangle+\int_{0}^{\tau}\left\langle w_{h}(t), \partial_{-h} u_{h}(t)\right\rangle \\
=\int_{0}^{\tau}\left\langle f_{h}(t), \partial_{-h} u_{h}(t)\right\rangle .
\end{gathered}
$$


In view of maximal monotonicity of subdifferentials, we have

$$
\int_{0}^{\tau}\left\langle\partial_{-h} v_{h}(t), \partial_{-h} u_{h}(t)\right\rangle \geq 0
$$

By virtue of Lemma 13, the second term in (35) could be estimated as follows:

$$
\begin{aligned}
\int_{0}^{\tau}\left\langle w_{h}(t), \partial_{-h} u_{h}(t)\right\rangle & \geq \int_{0}^{\tau} \partial_{-h} \Phi_{B}\left(u_{h}(t)\right) d t \\
& =\Phi_{B}\left(u_{h}(\tau)\right)-\Phi_{B}\left(u_{0}\right) .
\end{aligned}
$$

As for the third term, applying integration by parts, we have

$$
\begin{aligned}
\int_{0}^{\tau}\left\langle f_{h}(t), \partial_{-h} u_{h}(t)\right\rangle= & \left\langle f_{h}(\tau), u_{h}(\tau)\right\rangle-\left\langle f_{h}(h), u_{h}(0)\right\rangle \\
& -\int_{0}^{\tau-h}\left\langle\partial_{h} f_{h}(t), u_{h}(t)\right\rangle d t .
\end{aligned}
$$

Since $f_{h} \rightarrow f$ in $W^{1,2}\left(0, T ; V^{\prime}\right)$ as $h \rightarrow 0$ and the embedding $W^{1,2}\left(0, T ; V^{\prime}\right) \rightarrow C\left([0, T] ; V^{\prime}\right)$ is continuous, there exists a constant $C>0$ such that

$$
\int_{0}^{\tau}\left\langle f_{h}(t), \partial_{-h} u_{h}(t)\right\rangle \leq C+C \sup _{0 \leq t \leq T}\left\|u_{h}(t)\right\|_{V} .
$$

Then, from the assumption on initial value (see (V) Section 2.1), it follows that

$$
\sup _{0 \leq t \leq T} \Phi_{B}\left(u_{h}(t)\right) \leq C+C \sup _{0 \leq t \leq T}\left\|u_{h}(t)\right\|_{V} .
$$

From the coercivity of $\Phi_{B}$ (see (4)), we get (32) and (33) from (40) and (5). Applying $\partial_{-h} v_{h}(t)=f_{h}-w_{h}$, we get (34).

\subsection{Completion of Proof}

Lemma 19. There exist $u \in L^{\infty}(0, T ; V), v(t) \in L^{\infty}\left(0, T ; V^{\prime}\right) \cap$ $W^{1,2}\left(0, T ; V^{\prime}\right)$, and $w(t) \in L^{\infty}\left(0, T ; V^{\prime}\right)$ such that for almost all $t \in[0, T], v(t)=\mathscr{A}(u(t)), w(t) \in \mathscr{B}(u(t))$, and

$$
\frac{d}{d t} v(t)+w(t)=f(t) \quad \text { in } V^{\prime}
$$

Moreover, $v(t) \rightarrow v_{0}$ strongly in $V^{\prime}$ as $t \rightarrow 0$.

Proof. From (32) and (33), there exist $u \in L^{\infty}(0, T ; V)$ and $w \in L^{\infty}\left(0, T ; V^{\prime}\right)$ such that

$$
\begin{gathered}
u_{h}(t) \longrightarrow u \quad \text { weakly in } L^{2}(0, T ; V), \\
w_{h}(t) \longrightarrow w \quad \text { weakly in } L^{2}\left(0, T ; V^{\prime}\right) .
\end{gathered}
$$

Since $\mathscr{A}$ is compact from $V$ to $V^{\prime}$ (see Remark 16) and $u_{h}$ is uniformly bounded in $L^{\infty}(0, T ; V), v_{h}(t)$ is precompact in $V^{\prime}$ for each $t \in[0, T]$. Then combining (34), there exist a subsequence of $v_{h}$ (still denoted by $v_{h}$ ) and $v \in L^{\infty}\left(0, T ; V^{\prime}\right)$ such that

$$
v_{h}(t) \longrightarrow v \text { strongly in } C\left(0, T ; V^{\prime}\right) .
$$

In addition, from (34), there exists $\chi \in L^{\infty}\left(0, T ; V^{\prime}\right)$ such that

$$
\partial_{-h} v_{h}(t) \longrightarrow \chi \quad \text { weakly in } L^{2}\left(0, T ; V^{\prime}\right)
$$

and we can easily testify that $\chi=d v / d t$ combining (44).

In virtue of $(30)$, for any $x \in L^{2}(0, T ; V)$, we have

$$
\int_{0}^{T}\left\langle\partial_{-h} v_{h}(t), x\right\rangle d t+\int_{0}^{T}\left\langle w_{h}(t), x\right\rangle d t=\int_{0}^{T}\left\langle f_{h}(t), x\right\rangle d t .
$$

Applying (43) and (45) and letting $h \rightarrow 0$, it follows that

$$
\int_{0}^{T}\left\langle\frac{d}{d t} v(t), x\right\rangle d t+\int_{0}^{T}\langle w(t), x\rangle d t=\int_{0}^{T}\langle f(t), x\rangle d t
$$

which implies that for almost all $t \in(0, T]$,

$$
\frac{d}{d t} v(t)+w(t)=f(t) \text { in } V^{\prime}
$$

To complete the proof, we need to show that $v=\mathscr{A}(u)$, $w \in \mathscr{B}(u)$ for almost all $t \in[0, T]$. Since $\mathscr{A}, \mathscr{B}$ are maximal monotone operators, then, combining Lemmas 6 and 11, it suffices to prove that

$$
\begin{gathered}
\limsup _{h \rightarrow 0} \int_{0}^{T}\left\langle v_{h}(t), u_{h}(t)\right\rangle d t \leq \int_{0}^{T}\langle v(t), u(t)\rangle d t \\
\underset{h \rightarrow 0}{\limsup } \int_{0}^{T}\left\langle w_{h}(t), u_{h}(t)\right\rangle d t \leq \int_{0}^{T}\langle w(t), u(t)\rangle d t .
\end{gathered}
$$

In view of (42) and (44), we have

$$
\lim _{h \rightarrow 0} \int_{0}^{T}\left\langle v_{h}(t), u_{h}(t)\right\rangle d t=\int_{0}^{T}\langle v(t), u(t)\rangle d t
$$

which implies (49).

Applying (30), we have

$$
\begin{aligned}
\int_{0}^{T}\left\langle w_{h}(t), u_{h}(t)\right\rangle d t= & \int_{0}^{T}\left\langle f_{h}(t), u_{h}(t)\right\rangle d t \\
& -\int_{0}^{T}\left\langle\partial_{-h} v_{h}(t), u_{h}(t)\right\rangle d t .
\end{aligned}
$$

Since $f_{h} \rightarrow f$ strongly in $L^{2}\left(0, T ; V^{\prime}\right)$ and $u_{h} \rightarrow u$ weakly in $L^{2}(0, T ; V)$, we have

$$
\lim _{h \rightarrow 0} \int_{0}^{T}\left\langle f_{h}(t), u_{h}(t)\right\rangle d t=\int_{0}^{T}\langle f(t), u(t)\rangle d t
$$

For the second term, applying Lemma 13, we have

$$
\begin{gathered}
\liminf _{h \rightarrow 0} \int_{0}^{T}\left\langle\partial_{-h} v_{h}(t), u_{h}(t)\right\rangle d t \\
\quad \geq \liminf _{h \rightarrow 0} \int_{0}^{T} \partial_{-h} \Phi_{A}^{*}\left(v_{h}(t)\right) d t \\
\geq \Phi_{A}^{*}(v(T))-\Phi_{A}^{*}\left(v_{0}\right),
\end{gathered}
$$


since $v_{h}(T) \rightarrow v(T)$ strongly in $V^{\prime}$ and $\Phi_{A}^{*}$ is lower semicontinuous. Moreover, from Lemma 14 , we can easily get

$$
\Phi_{A}^{*}(v(T))-\Phi_{A}^{*}\left(v_{0}\right)=\int_{0}^{T}\left\langle\frac{d}{d t} v(t), u(t)\right\rangle d t .
$$

Therefore,

$$
\begin{aligned}
& \limsup _{h \rightarrow 0} \int_{0}^{T}\left\langle w_{h}(t), u_{h}(t)\right\rangle d t \\
& \quad \leq \int_{0}^{T}\left\langle f(t)-\frac{d}{d t} v(t), u(t)\right\rangle d t .
\end{aligned}
$$

On the other hand, applying (47) on $u$, we have

$$
\int_{0}^{T}\left\langle f(t)-\frac{d}{d t} v(t), u(t)\right\rangle d t=\int_{0}^{T}\langle w(t), u(t)\rangle d t
$$

Consequently, we obtain (50).

As the end of the proof, since $v_{h} \rightarrow v$ in $C\left(0, T ; V^{\prime}\right)$ and

$$
\left\|v_{h}(t)-v_{0}\right\|_{V^{\prime}} \leq \int_{0}^{t}\left\|\partial_{-h} v_{h}(\tau)\right\|_{V^{\prime}} d \tau \leq C \sqrt{t} \longrightarrow 0
$$

as $t \rightarrow 0$, we have $v(t) \rightarrow v_{0}$ strongly in $V^{\prime}$ as $t \rightarrow 0$.

\section{Application to an Initial Boundary Value Problem}

The abstract existence can be applied to many models in fluid mechanics (see $[26,27]$ ). We shall illustrate the application of Theorem 2 to establish the existence of a solution to a nonlinear parabolic initial-boundary-value problem with nonlinear degenerate terms under the time derivative. This problem includes a nonlinear dynamic boundary condition.

Let $\Omega$ be a bounded domain in $\mathbb{R}^{3}$ with smooth boundary $\Gamma=\partial \Omega$ and $\Gamma$ has the partition $\Gamma=\Gamma_{1} \cup \Gamma_{2}$. Consider

$$
\begin{gathered}
\frac{\partial}{\partial t} a(u)-\sum_{i=1}^{3} \frac{\partial}{\partial x_{i}}\left(|\nabla u|^{p-2} \frac{\partial u}{\partial x_{i}}\right)=f(x, t), \\
(x, t) \in \Omega \times(0, T], \\
\frac{\partial}{\partial t} c(u)+|\nabla u|^{p-2} \nabla u \cdot n+g(x)|u|^{r-2} u=0, \\
(x, t) \in \Gamma_{1} \times(0, T], \\
u=0, \quad(x, t) \in \Gamma_{2} \times(0, T], \\
a(u)=a_{0}(x), \quad(x, t) \in \Omega \times\{0\}, \\
c(u)=c_{0}(x), \quad(x, t) \in \Gamma_{1} \times\{0\},
\end{gathered}
$$

where $2 \leq p<+\infty, 1 \leq r \leq p, a(\cdot)$ and $c(\cdot)$ are continuous and nondecreasing and $g(x) \in L^{\infty}(\Omega)$ with $g(x) \geq g_{0}>0$.

Remark 20. For the case meas $\left(\Gamma_{1}\right)>0$, the problem (59) cannot be covered by [8].
5.1. Formulation of Abstract Form. Let $H=L^{2}(\Omega) \oplus L^{2}\left(\Gamma_{1}\right)$ and $V=\left\{u \in W^{1, p}(\Omega): u=0\right.$ on $\left.\Gamma_{2}\right\}$, equipped with the norms $\|u\|_{V}=\|\nabla u\|_{L^{p}(\Omega)}+\|u\|_{L^{p}\left(\Gamma_{1}\right)}$ and $\left\|\left(u_{1}, u_{2}\right)\right\|_{H}=$ $\left\|u_{1}\right\|_{L^{2}(\Omega)}+\left\|u_{2}\right\|_{L^{2}\left(\Gamma_{1}\right)}$, respectively. Then $V$ is embedded in $H$ densely and compactly, and denote the injection by $i$. Assume that $a(\cdot), c(\cdot): \mathbb{R} \rightarrow \mathbb{R}$ are nondecreasing and continuous, and $\phi_{a}, \phi_{c}: \mathbb{R} \rightarrow(-\infty,+\infty]$ satisfy

$$
\phi_{a}(x)=\int_{0}^{x} a(s) d s, \quad \phi_{c}(x)=\int_{0}^{x} c(s) d s .
$$

Define

$$
\begin{gathered}
\Phi_{a}(u)=\int_{\Omega} \phi_{a}(u) d x, \quad \forall u \in L^{2}(\Omega), \\
\Phi_{c}(u)=\int_{\Gamma_{1}} \phi_{c}(u) d s, \quad \forall u \in L^{2}\left(\Gamma_{1}\right), \\
\Phi_{A}(u)=\Phi_{a}\left(u_{1}\right)+\Phi_{c}\left(u_{2}\right), \quad \forall u=\left(u_{1}, u_{2}\right) \in H, \\
\Phi_{B}(u)=\frac{1}{p} \int_{\Omega}|\nabla u|^{p} d x+\frac{1}{r} \int_{\Gamma_{1}} g(x)|u|^{r} d s, \quad \forall u \in V . \\
\text { Let } \mathscr{A}=\partial_{V}\left(\Phi_{A} \circ i\right) \text { and } \mathscr{B}=\partial_{V} \Phi_{B} ; \text { then } \forall v \in V, \\
\langle\mathscr{A}(u), v\rangle=\int_{\Omega} a(u) v d x+\int_{\Gamma_{1}} c(u) v d s, \\
\langle\mathscr{B}(u), v\rangle=\int_{\Omega}|\nabla u|^{p-2} \nabla u \nabla v d x+\int_{\Gamma_{1}} g(x)|u|^{r-2} u v d s, \\
\langle F(t), v\rangle=\int_{\Omega} f(x, t) v d x .
\end{gathered}
$$

Assuming $a_{0} \in L^{2}(\Omega)$ and $v_{0} \in L^{2}\left(\Gamma_{1}\right)$, the problem (59) can be rewritten as

$$
(\mathrm{CP}) \begin{cases}\frac{d}{d t} \mathscr{A}(u)+\mathscr{B}(u) \ni F(t) & t \in(0, T), \\ \mathscr{A}(u) \ni v_{0} & t=0,\end{cases}
$$

where $v_{0}=\left(a_{0}, c_{0}\right) \in H \subset V^{\prime}$.

5.2. Existence of Solutions. Applying the existence theorem of abstract form, we could claim the solvability of the problem (59), by some appropriate assumptions.

$(\mathrm{H} 1) a(\cdot), c(\cdot): \mathbb{R} \rightarrow \mathbb{R}$ are nondecreasing and continuous.

(H2) $\forall\left(u_{1}, u_{2}\right) \in H=L^{2}(\Omega) \oplus L^{2}\left(\Gamma_{1}\right), a\left(u_{1}\right) \in L^{2}(\Omega)$, and $c\left(u_{2}\right) \in L^{2}\left(\Gamma_{1}\right)$

(H3) $g \in L^{\infty}\left(\Gamma_{1}\right)$ with $g \geq g_{0}>0$.

Assume that (H1)-(H3) hold; then $\Phi_{A}$ and $\Phi_{B}$ satisfy Theorem 2 . Therefore, for any $v_{0}$ and $F$ satisfying Theorem 2 , the abstract problem (63) is solvable, which implies the existence for the solution of (59) as follows. 
Theorem 21. Assume (H1)-(H3). Let $2 \leq p<+\infty, 1 \leq r \leq p$, and $p^{\prime}=p /(p-1)$. Assume that $f \in W^{1,2}\left(0, T ; W^{-1, p^{\prime}}(\Omega)\right)$. Assume $\left(a_{0}, c_{0}\right) \in L^{2}(\Omega) \oplus L^{2}\left(\Gamma_{1}\right)$ satisfying

$$
\begin{gathered}
\exists u_{0} \in\left\{u \in W^{1, p}(\Omega): u=0 \text { on } \Gamma_{2}\right\} \text { such that } \\
a\left(u_{0}\right)=a_{0}, \quad c\left(u_{0}\right)=c_{0} .
\end{gathered}
$$

Then there exists at least one solution $u(x, t)$ of (59); that is, $u$ is a measurable function such that

$$
\begin{gathered}
u \in L^{\infty}\left(0, T ; W^{1, p}(\Omega)\right), \\
a(u) \in L^{\infty}\left(0, T ; W^{-1, p^{\prime}}(\Omega)\right) \cap W^{1,2}\left(0, T ; W^{-1, p^{\prime}}(\Omega)\right), \\
c(u) \in L^{\infty}\left(0, T ; L^{p^{\prime}}\left(\Gamma_{1}\right)\right) \cap W^{1,2}\left(0, T ; L^{p^{\prime}}\left(\Gamma_{1}\right)\right),
\end{gathered}
$$

and for a. e. $t \in(0, T)$,

$$
\begin{aligned}
\int_{\Omega} \frac{\partial}{\partial t} & a(u) \phi d x+\int_{\Omega}|\nabla u|^{p-2} \nabla u \cdot \nabla \phi d x \\
& +\int_{\Gamma_{1}}\left(\frac{\partial}{\partial t} c(u)+g(x)|u|^{p-2} u\right) \phi d s \\
= & \int_{\Omega} f(x, t) \phi d x
\end{aligned}
$$

for any $\phi \in W^{1, p}(\Omega)$. Moreover, $a(u(t)) \rightarrow a_{0}$ strongly in $W^{-1, p^{\prime}}(\Omega)$ and $c(u(t)) \rightarrow c_{0}$ strongly in $L^{p^{\prime}}\left(\Gamma_{1}\right)$ as $t \rightarrow 0$.

Remark 22. More generally, instead of the boundary condition on $\Gamma_{1}$ in the problem (59), we assume that (see [27])

$$
\begin{array}{r}
\frac{\partial}{\partial t} c(u)+|\nabla u|^{p-2} \nabla u \cdot n+b(u)+g(x)|u|^{r-2} u \ni 0, \\
(x, t) \in \Gamma_{1} \times(0, T],
\end{array}
$$

where $b(\cdot)$ is multivalued and maximal monotone. Assume that there exists a proper, convex, lower semicontinuous function $\phi_{b}$ defined on $\mathbb{R}$ with $\phi_{b}(0)=0$ such that $b(\cdot)=$ $\partial \phi_{b}(\cdot)$. Define

$$
\Phi_{b}(u)=\int_{\Gamma_{1}} \phi_{b}(u) d s \quad \forall u \in V,
$$

and make a modification of $\Phi_{B}$ as follows:

$$
\Phi_{B}(u)=\frac{1}{p} \int_{\Omega}|\nabla u|^{p} d x+\frac{1}{r} \int_{\Gamma_{1}} g(x)|u|^{r} d s+\Phi_{b}(u)
$$

$\forall u \in V$.

Then the problem can be solved by imposing some appropriate assumptions on $b$. For a simple case, we could suppose that

(b1) there exists a constant $b_{0} \in \mathbb{R}$, such that $y \geq b_{0}$ for any $x \in \mathbb{R}$, and $y \in b(x)$; (b2) there exists a nondecreasing function $l_{b}(\cdot): \mathbb{R} \rightarrow \mathbb{R}$ such that

$$
\|b(u)\|_{L^{p^{\prime}}\left(\Gamma_{1}\right)} \leq l_{b}\left(\Phi_{b}(u)\right), \quad \forall u \in L^{p}\left(\Gamma_{1}\right) .
$$

Then $\Phi_{B}$ satisfy Theorem 2. In addition, (b1) and (b2) can be satisfied by extensive functions. For example, for $r_{0} \in \mathbb{R}$, set

$$
b(s)= \begin{cases}1, & s>r_{0} \\ {[-1,1],} & s=r_{0} \\ -1, & s<r_{0}\end{cases}
$$

and $\Phi_{b}(u)=\int_{\Gamma_{1}}\left|u-r_{0}\right| d s$.

\section{Conflict of Interests}

The authors declare that there is no conflict of interests regarding the publication of this paper.

\section{Acknowledgment}

This research was supported partially by NSFC under Grant no. 11271218 .

\section{References}

[1] G. Akagi, "Doubly nonlinear evolution equations and Bean's critical-state model for type-II superconductivity," Discrete and Continuous Dynamical Systems A, pp. 30-39, 2005.

[2] G. Akagi and M. Ôtani, "Evolution inclusions governed by subdifferentials in reflexive Banach spaces," Journal of Evolution Equations, vol. 4, no. 4, pp. 519-541, 2004.

[3] R. E. Showalter, Monotone Operators in Banach Space and Nonlinear Partial Differential Equations, vol. 49, American Mathematical Society, Providence, RI, USA, 1997.

[4] R. E. Showalter and B. Momken, "Single-phase flow in composite poroelastic media," Mathematical Methods in the Applied Sciences, vol. 25, no. 2, pp. 115-139, 2002.

[5] R. E. Showalter and U. Stefanelli, "Diffusion in poro-plastic media," Mathematical Methods in the Applied Sciences, vol. 27, no. 18, pp. 2131-2151, 2004.

[6] S. Aizicovici and V.-M. Hokkanen, "Doubly nonlinear equations with unbounded operators," Nonlinear Analysis: Theory, Methods \& Applications, vol. 58, no. 5-6, pp. 591-607, 2004.

[7] G. Akagi, "Doubly nonlinear evolution equations governed by time-dependent subdifferentials in reflexive Banach spaces," Journal of Differential Equations, vol. 231, no. 1, pp. 32-56, 2006.

[8] H. W. Alt and S. Luckhaus, "Quasilinear elliptic-parabolic differential equations," Mathematische Zeitschrift, vol. 183, no. 3, pp. 311-341, 1983.

[9] V. Barbu, "Existence for nonlinear Volterra equations in Hilbert spaces," SIAM Journal on Mathematical Analysis, vol. 10, no. 3, pp. 552-569, 1979.

[10] E. DiBenedetto and R. E. Showalter, "Implicit degenerate evolution equations and applications," SIAM Journal on Mathematical Analysis, vol. 12, no. 5, pp. 731-751, 1981.

[11] N. Kenmochi, "Solvability of nonlinear evolution equations with time-dependent constraints and applications," Bulletin of the Faculty of Education. Chiba University, vol. 30, pp. 1-87, 1981. 
[12] N. Kenmochi and I. Pawłow, "A class of nonlinear ellipticparabolic equations with time-dependent constraints," Nonlinear Analysis: Theory, Methods \& Applications, vol. 10, no. 11, pp. 1181-1202, 1986.

[13] M. Kubo, "Characterization of a class of evolution operators generated by time-dependent subdifferentials," Funkcialaj Ekvacioj, vol. 32, no. 2, pp. 301-321, 1989.

[14] R. E. Showalter and N. Su, "Partially saturated flow in a poroelastic medium," Discrete and Continuous Dynamical Systems B, vol. 1, no. 4, pp. 403-420, 2001.

[15] Y. Yamada, "On evolution equations generated by subdifferential operators," Journal of the Faculty of Science. University of Tokyo, vol. 23, no. 3, pp. 491-515, 1976.

[16] S. Yotsutani, "Evolution equations associated with the subdifferentials," Journal of the Mathematical Society of Japan, vol. 31, no. 4, pp. 623-646, 1979.

[17] F. Otto, " $L$-contraction and uniqueness for quasilinear ellipticparabolic equations," Journal of Differential Equations, vol. 131, no. 1, pp. 20-38, 1996.

[18] Y. Alber and I. Râzanceva, Nonlinear Ill-Posed Problems of Monotone Type, Springer, 2006.

[19] V. Barbu, Nonlinear Semigroups and Differential Equations in Banach Spaces, P. Noordhoff, Leyden, The Netherlands, 1976.

[20] V. Barbu, Nonlinear Differential Equations of Monotone Types in Banach Spaces, Springer, New York, NY, USA, 2010.

[21] H. Brézis, Opérateurs Maximaux Monotones et Semi-Groupes de Contractions dans les Espaces de Hilbert, North-Holland Publishing, Amsterdam, The Netherlands, North-Holland Mathematics Studies, 1973.

[22] R. S. Burachik and A. N. Iusem, Set-Valued Mappings and Enlargements of Monotone Operators, vol. 8, Springer, New York, NY, USA, 2008.

[23] G. Duvaut and J.-L. Lions, Inequalities in Mechanics and Physics, Springer, Berlin, Germany, 1976.

[24] N. Kenmochi, "Some nonlinear parabolic variational inequalities," Israel Journal of Mathematics, vol. 22, no. 3-4, pp. 304-331, 1975.

[25] U. Stefanelli, "The Brezis-Ekeland principle for doubly nonlinear equations," SIAM Journal on Control and Optimization, vol. 47, no. 3, pp. 1615-1642, 2008.

[26] I. Borsi, A. Farina, and A. Fasano, "On the infiltration of rain water through the soil with runoff of the excess water," Nonlinear Analysis: Real World Applications, vol. 5, no. 5, pp. 763-800, 2004.

[27] I. Borsi, A. Farina, and M. Primicerio, "A rain water infiltration model with unilateral boundary condition: qualitative analysis and numerical simulations," Mathematical Methods in the Applied Sciences, vol. 29, no. 17, pp. 2047-2077, 2006. 


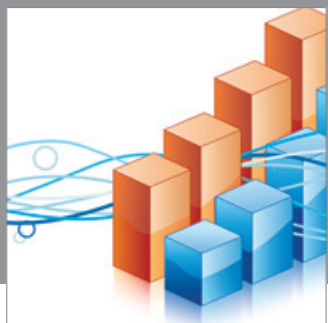

Advances in

Operations Research

mansans

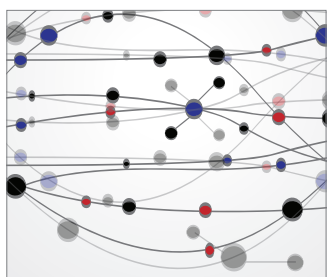

The Scientific World Journal
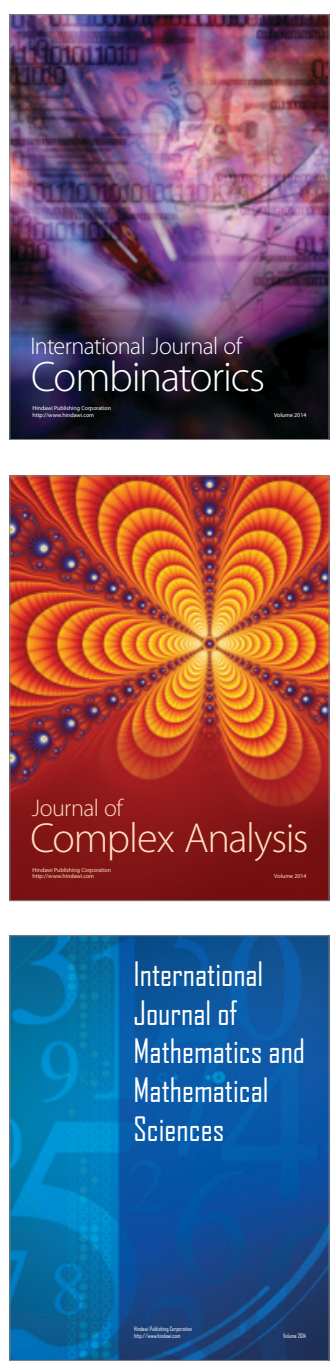
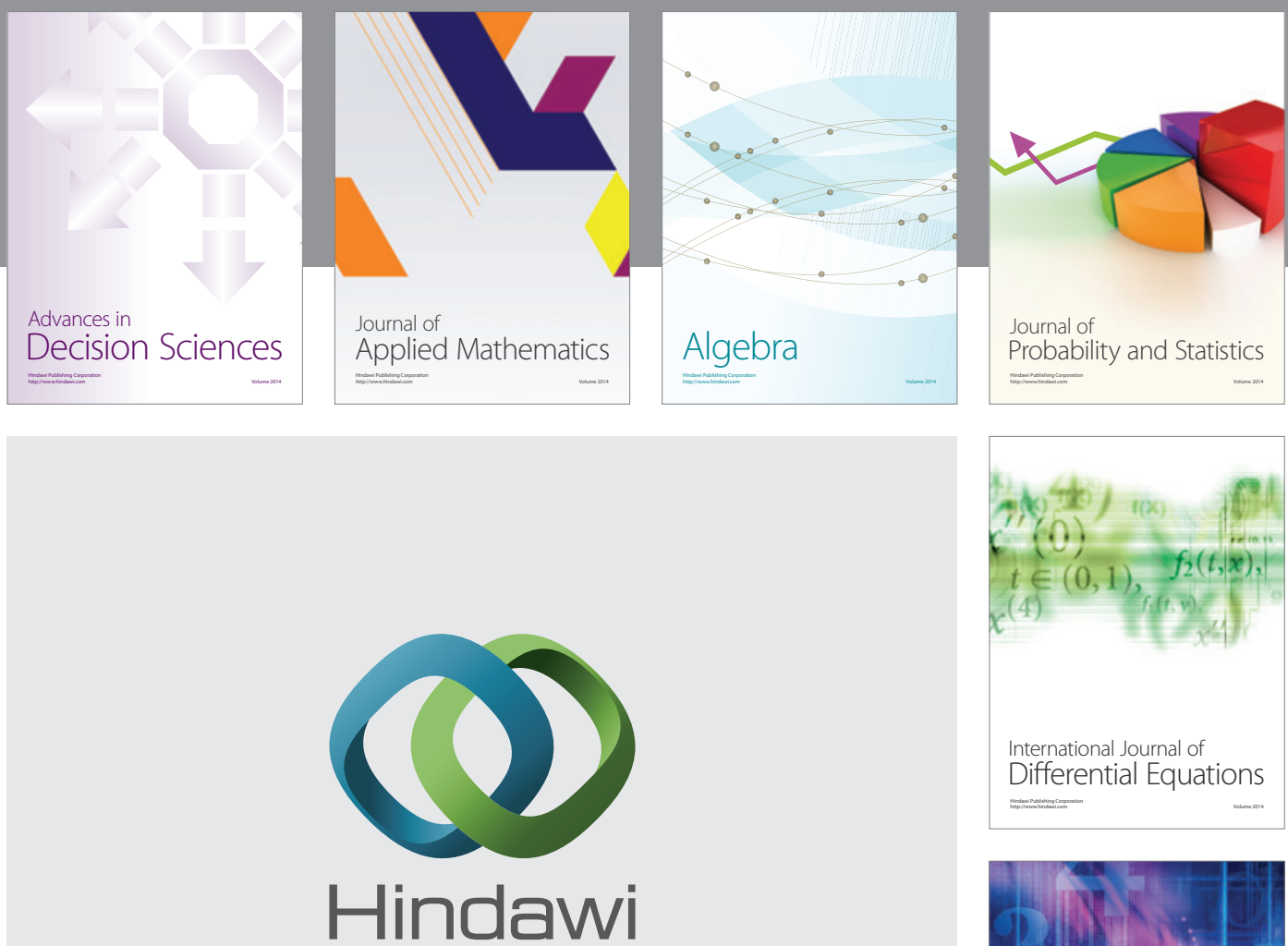

Submit your manuscripts at http://www.hindawi.com
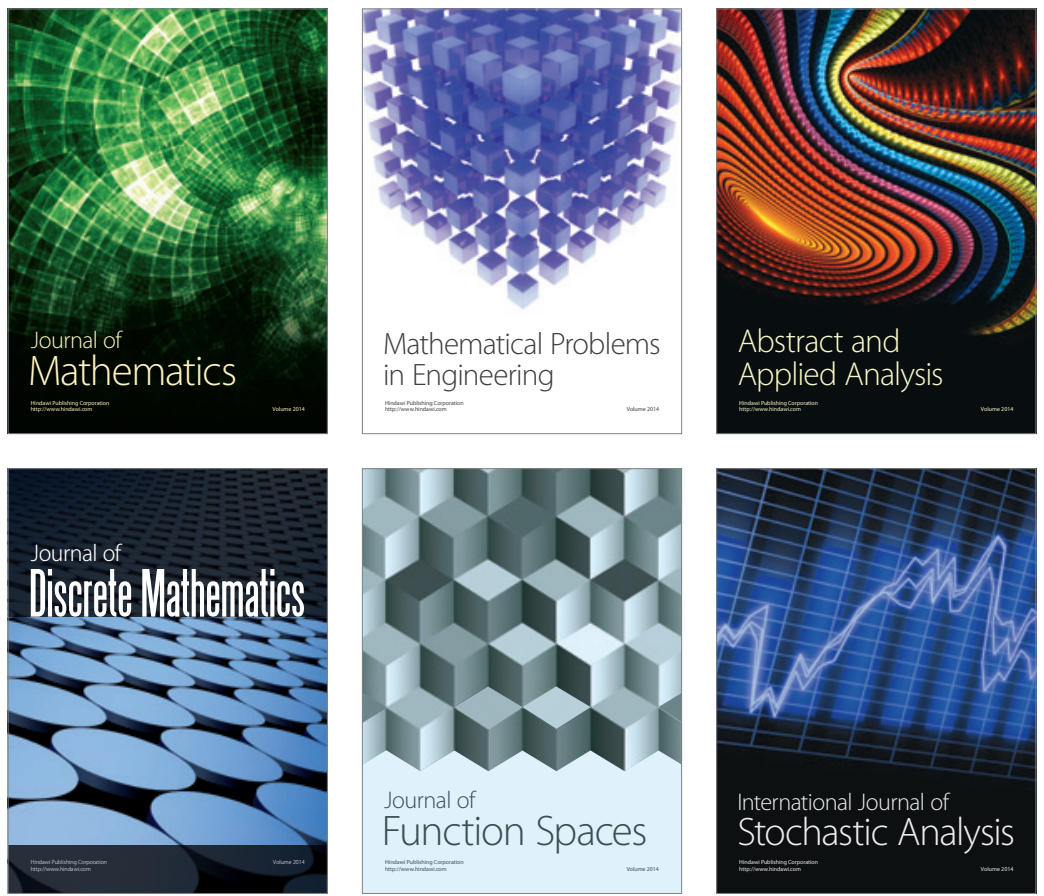

Journal of

Function Spaces

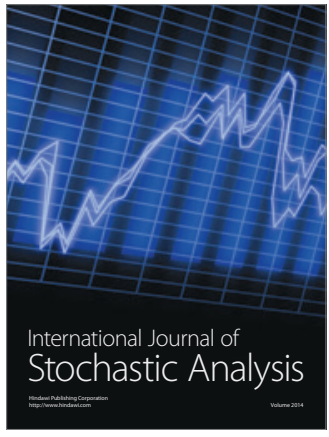

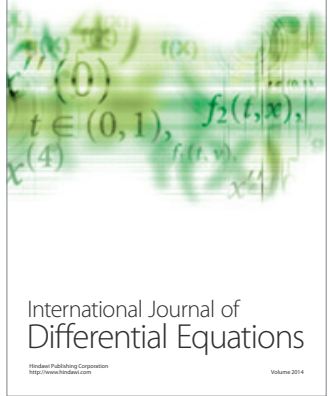
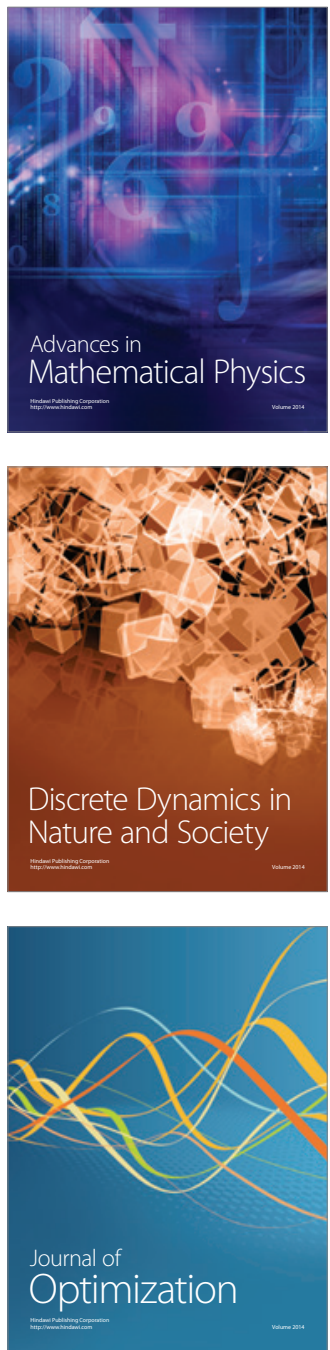